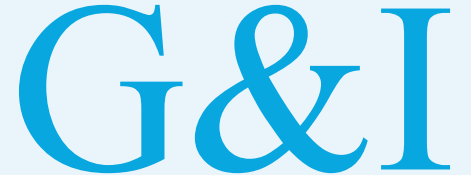

Genomics \& INFORMatics

\section{Original article}

eISSN 2234-0742

Genomics Inform 2021;19(3):e29

https://doi.org/10.5808/gi.21034

Received: June 14, 2021

Revised: July 16, 2021

Accepted: July 22, 2021

*Corresponding author:

E-mail: reddyg.bp@icmr.gov.in

${ }^{*}$ Corresponding author:

E-mail:drdirisala@gmail.com

\title{
Molecular insights into the role of genetic determinants of congenital hypothyroidism
}

\author{
Yedukondalu Kollati', Radha Rama Devi Akella ${ }^{2,3}$, \\ Shaik Mohammad Naushad ${ }^{3}$, Rajesh K. Patel ${ }^{4}$, \\ G. Bhanuprakash Reddy ${ }^{5^{*}}$, Vijaya R. Dirisala ${ }^{1 *}$
}

'Department of Biotechnology, Vignan's University, Vadlamudi, Guntur, Andhra Pradesh 522213, India

${ }^{2}$ Department of Genetics, Rainbow Children's Hospital, Banjara Hills, Hyderabad, Telangana 500009, India

${ }^{3}$ Department of Biochemical Genetics and Pharmacogenomics, Sandor Speciality Diagnostics Pvt. Ltd, Banjara Hills, Hyderabad, Telangana 500034, India

${ }^{4}$ Department of Genetics, Genetic Group of Gujarat Diagnostic Centre, Mehsana, Gujarat 384002, India

${ }^{5}$ Biochemistry Division, National Institute of Nutrition, Hyderabad, Telangana 500007, India

In our previous studies, we have demonstrated the association of certain variants of the thyroid-stimulating hormone receptor (TSHR), thyroid peroxidase (TPO), and thyroglobulin (TG) genes with congenital hypothyroidism. Herein, we explored the mechanistic basis for this association using different in silico tools. The mRNA 3'-untranslated region (3'-UTR) plays key roles in gene expression at the post-transcriptional level. In TSHR variants (rs2268477, rs7144481, and rs17630128), the binding affinity of microRNAs (miRs) (hsamiR-154-5p, hsa-miR-376a-2-5p, hsa-miR-3935, hsa-miR-4280, and hsa-miR-6858-3p) to the 3'-UTR is disrupted, affecting post-transcriptional gene regulation. TPO and TG are the two key proteins necessary for the biosynthesis of thyroid hormones in the presence of iodide and $\mathrm{H}_{2} \mathrm{O}_{2}$. Reduced stability of these proteins leads to aberrant biosynthesis of thyroid hormones. Compared to the wild-type TPO protein, the p.S398T variant was found to exhibit less stability and significant rearrangements of intra-atomic bonds affecting the stoichiometry and substrate binding (binding energies, $\Delta \mathrm{G}$ of wild-type vs. mutant: $-15 \mathrm{vs}$. $-13.8 \mathrm{kcal} / \mathrm{mol}$; and dissociation constant, $\mathrm{K}_{d}$ of wild-type vs. mutant: $7.2 \mathrm{E}^{-12} \mathrm{vs} .7 .0 \mathrm{E}^{-11} \mathrm{M}$ ). The missense mutations p.G653D and p.R1999W on the TG protein showed altered $\Delta G$ $(0.24 \mathrm{kcal} / \mathrm{mol}$ and $0.79 \mathrm{kcal} / \mathrm{mol}$, respectively). In conclusion, an in silico analysis of TSHR genetic variants in the 3'-UTR showed that they alter the binding affinities of different miRs. The TPO protein structure and mutant protein complex (p.S398T) are less stable, with potentially deleterious effects. A structural and energy analysis showed that TG mutations (p.G653D and p.R1999W) reduce the stability of the TG protein and affect its structure-functional relationship.

Keywords: congenital hypothyroidism, miR, TG, TPO, TSHR, 3'-UTR

\section{Introduction}

Congenital hypothyroidism $(\mathrm{CH})$ is one of the most common endocrine disorders, reported to occur in 1 in 3,000 to 4,000 newborns worldwide [1,2], and 1 in 1,100 in India [3]. On a worldwide basis, $\mathrm{CH}$ frequently results from iodine deficiency. Otherwise, $\mathrm{CH}$ is commonly caused by thyroid gland development defects, which can lead to thyroid dys- 
genesis $(80 \%-85 \%)$ [1]. The majority of these cases involve thyroid dysgenesis [4], agenesis (35\%-40\%), ectopic tissue (30\%$45 \%)$, or hypoplasia (5\%) [1]. Thyroid dysgenesis is caused by genes (thyroid transcription factor-1 [TTF-1], thyroid transcription factor-2 [TTF-2], and paired box 8 [PAX-8]) associated with syndromic $\mathrm{CH}$ and those causing non-syndromic $\mathrm{CH}$ (thyroid-stimulating hormone receptor [TSHR]) [5]. The remaining 15\%-20\% of cases are due to hereditary defects in the genes involved in the intermediary steps of biosynthesis in the thyroid, leading to dyshormonogenesis [6]. Thyroid dyshormonogenesis is associated with multiple genetic defects, including dual oxidases (DUOXs; DUOX1 and DUOX2), and its maturation factors (DUOXA1 and DUOXA2), thyroid peroxidase (TPO), thyroglobulin (TG), dehalogenase 1 (DEHAL1) and solute carrier families: 26 (SLC26A4 or PDS) and 5 (SLC5A5 or NIS) [1,4]. The synthesis of thyroid hormones $\left(\mathrm{T}_{4}\right.$ and $\left.\mathrm{T}_{3}\right)$ is affected in $20 \%$ of all cases involving inborn genetic errors in the enzymatic cascade, which is defined as thyroid dyshormonogenesis [1]. Most often, these defects appear to be transmitted in an autosomal recessive manner [6], but autosomal dominant inheritance has also been reported [7].

Splicing is dependent on the exact identification of exons, which are perfectly recognized within pre-mRNAs. The presence of 5' and $3^{\prime}$ splice sites and the branch points may not be sufficient to define intron-exon boundaries. The exonic elements are represented by exonic splicing enhancers (ESEs), where SR proteins bind and play a pivotal role in spliceosome assembly. Sequences that act as exonic splicing silencers (ESSs) bind to negative regulators, which belong to the heterogeneous nuclear ribonucleoprotein family. Both ESEs and ESSs appear to play an instrumental role in the regulation of alternative splicing events, other than the sequences that may play a pertinent role in the definition of constitutive exons [8].

Furthermore, microRNAs (miRs), which are 22-23 nucleotides in length, bind to the $3^{\prime}$-untranslated region ( $3^{\prime}$-UTR) and regulate mRNAs post-transcriptionally, either by facilitating mRNA degradation or by inhibiting mRNA transcription [9]. More than 30\% of genes encoding for proteins are regulated by miRs [10]. Any genetic variation in the $3^{\prime}$-UTR may interfere with miR binding to its target, thereby influencing the expression of the targeted gene [11].

In our previous study, we investigated the effects of TSHR, TPO, and $T G$ genetic variants in $\mathrm{CH}$ and identified 22 variants [12]. Three of these 22 variants (p.S398T in TPO and p.G653D and p. $\mathrm{R} 1999 \mathrm{~W}$ in $T G$ ) were predicted to be deleterious [12]. In this study, we aimed to perform an in silico analysis to achieve a better understanding of polymorphic variants in the 3'-UTR of the TSHR gene, which interferes with the binding affinities of miRs that might inhibit gene expression. Further, we analyzed mutant protein structure-function relationships through molecular modeling and inter- action studies for the p.S398T mutation of TPO. In addition, molecular modeling, mutation analyses, and thermodynamic energy calculations for variants of p.G653D and p.R1999W in the TG gene were also performed.

\section{Methods}

As part of a newborn screening (NBS) program for $\mathrm{CH}$, we analyzed 49,432 newborns, of whom 1,099 were screened with negative findings, along with 45 confirmed cases of $\mathrm{CH}$. The institutional ethical committee of Rainbow Children's Hospital, located in Hyderabad, India, approved the study protocol $(\mathrm{RCH}-$ $\mathrm{BH} / 066 / 02-2018)$. Informed consent was obtained from the parents or guardians of all neonates [12].

\section{In silico analysis of SNP-miR interactions}

Four web-based tools-PolymiRTS (http://compbio.uthsc.edu/ miRSNP/) [13], miRDB (http://www.mirdb.org/cgi-bin/ search. cgi) [14], TargetScan (http://www.targetscan.org) [15], and STarMir (http://sfold.wadsworth.org) [16]—were used to ascertain whether the identified single-nucleotide polymorphisms (SNPs) in the 3'-UTR region interfered with miR binding.

\section{Molecular modeling and protein-protein docking of the TPO protein}

To further analyze the predicted role of SNPs in the protein interactions, molecular modeling and interaction studies were carried out. The Uniprot IDs of TPO and DUOX1 are P07202 and Q9NRD9, respectively. As the structures of TPO and DUOX1 are not crystallized, model prediction for the functional domains of the proteins was carried using the I-Tasser server [17]. The TPO domain (residues 167-734) of human TPO was modeled, as was the similarly interacting protein of the human dual peroxidase domain of DUOX. The 398 Ser-Thr mutant of TPO was modeled and energy-minimized using Chimera [18], and energy minimization was carried out using the steepest descent method. The modeled structures were docked using the ClusPro server [19] and the probable interactions were predicted using the PIC webserver [20]. The protein-protein interaction energies were calculated using the PRODIGY server [21].

\section{Molecular modeling of the wild-type and mutant TG protein}

To understand the effect of SNPs such as p.G653D and p.R1999W on the TG protein, we performed molecular modeling, mutation analyses, and thermodynamic energy calculations using the SAAMBE-3D server [22]. The crystal structure of human TG was taken from the protein database (PDB ID: 6SCJ). The crystal structure 
was solved using electron microscopy with a resolution of $3.60 \AA$ [23]. Mutations such as p.G653D and p.R1999W were inserted in silico into the wild-type TG protein using the 'Mutagenesis' wizard of the 'PyMol' software [24]. These modeled structures of mutant TG proteins were further used to understand the effects of the mutations on protein structural stability and its intramolecular interactions. The effect of mutations such as p.G653D and p.R1999W on TG protein stability were checked using the SAAMBE-3D server [22], which predicts the effects of mutations on protein stability. In addition, we used the DynaMut server [25] to understand the effects of mutations on various types of interactions, such as van der Waals, weak polar van der Waals, polar proximal, amide-amide interactions, and so on.

\section{Results and Discussion}

In our previous studies, we established the reference intervals for thyroid-stimulating hormone (TSH) through NBS data, and the specific genotype-phenotype correlations were exhibited in confirmed $\mathrm{CH}$ cases with TSHR, TPO, and TG gene variants [12]. Previously, we published an in silico analysis on p.D727E in the TSHR gene, which might control the signal transduction (cAMP-mediated) pathway, consequently contributing to the pathophysiology of $\mathrm{CH}$ [26]. In this study, we specifically focused on an in silico analysis of three variants: p.S398T in TPO, and p.G653D and p.R1999W in TG.

In a study we reported earlier, we identified eight intronic variants (g. IVS 01+63 G>C, g.IVS 06-69 C > T, g.IVS 06+13 A > G, g.IVS $09+58 \mathrm{~T}>\mathrm{G}$ in the TSHR gene, g.IVS $11+20 \mathrm{G}>\mathrm{A}$, g.IVS $13+128 \mathrm{C}>\mathrm{T}$, g.IVS $14-37 \mathrm{G}>\mathrm{A}$, g.IVS $14-19 \mathrm{G}>\mathrm{C}$ in the TPO gene) [12]. The interpretation of the intronic variants is that g.IVS 01+63 G > C, g.IVS 06-69 C > T, g.IVS 09+58 T > C are involved in the creation of an intronic ESE site and g.IVS $06+13 \mathrm{~A}>\mathrm{G}$ is an alteration of an intronic ESS site. The intronic variant g.IVS $11+20$
G > A predicted a signal wherein an ESS site is broken and a new ESS site is created; this is interpreted as involving an alteration of an intronic ESS site and creation of an intronic ESE site. These results may not have an impact on splicing; no significant splicing motif alterations were detected for the remaining SNPs, which probably have no impact on the splicing mechanism (http://www.umd.be/ HSF3/HSF.shtml).

\section{In silico analysis of SNP-miR interaction}

The four SNPs in the 3'-UTR of the TSHR gene (rs2268477, rs373305430, rs7144481, and rs17630128) were predicted to alter the binding of 10 common miRs as per the data obtained from four different databases (PolymiRTS, miRDB, TargetScan, and STarMir). As shown in Table 1, the presence of the rs2268477 polymorphic variant destroys the binding site for hsa-miR-154-5p. The rs373305430, rs7144481, and rs17630128 polymorphic variants alter the binding affinities of hsa-miR-1237-5p, hsa-miR-4488, hsa-miR-4697-5p, hsa-miR-6846-5p, hsa-miR-6848-5p, hsa-miR376a-2-5p, hsa-miR-3935, hsa-miR-4280, and hsa-miR-6858-3p. The 3'-UTR SNPs-miR interaction hybrid diagrams are shown in Supplementary Fig. 1.

The rs2268477, rs373305430, rs7144481, and rs17630128 SNPs were localized in the 3 '-UTR region of the TSHR gene and hence associated with binding of the 10 different miRs. These SNPs in the miR target site on the 3'-UTR may affect the binding efficacy of $\mathrm{miR}$. SNPs may alter target gene expression to affect post-transcriptional processing and polyadenylation, and they may even contribute to $\mathrm{CH}$. Among the risk variants, rs2268477 was found to destroy the binding site of hsa-miR-154-5p. In papillary thyroid carcinoma, hsa-miR-154 was reported to be downregulated [27], which substantiates the role of miR-154 in thyroid development. The rs7144481 variant was found to alter binding affinities of hsa-miR376a-2-5p, hsa-miR-3935 and miR-4280. Long non-coding RNA

Table 1. In silico studies revealing TSHR SNP-miRNA interactions

\begin{tabular}{|c|c|c|c|c|c|c|c|}
\hline Gene & SNP & miRNA & Seed match & Wild & $\Delta \mathrm{G}(\mathrm{kcal} / \mathrm{mol})$ & Mutant & $\Delta \mathrm{G}(\mathrm{kcal} / \mathrm{mol})$ \\
\hline \multirow[t]{8}{*}{ TSHR } & rs2268477 & hsa-miR-154-5p & 7-mer & uauUAACCUAa & -22.0 & \multicolumn{2}{|c|}{ Disruption of binding site } \\
\hline & rs373305430 & hsa-miR-1237-5p & 6-mer & auuGCCCCCa & -23.4 & auuUCCCCCa & -17.4 \\
\hline & & hsa-miR-4697-5p & 6-mer & auuGCCCCCa & -29.8 & auuUCCCCCa & -22.8 \\
\hline & & hsa-miR-6846-5p & 6-mer & auuGCCCCCa & -29.6 & auuUCCCCCa & -17.3 \\
\hline & & hsa-miR-6848-5p & 6-mer & auuGCCCCCa & -26.3 & auuUCCCCCaа & -22.0 \\
\hline & & hsa-miR-3935 & 7-mer & auaAUCUACAc & -18.7 & auaAUCUAUAc & -14.0 \\
\hline & & hsa-miR-4280 & 7-mer & aauCUACACUa & -19.6 & aauCUAUACUa & -17.7 \\
\hline & rs17630128 & hsa-miR-6858-3p & 7-mer & cacGUUGGCUc & -20.2 & cacGCUGGCUc & -22.6 \\
\hline
\end{tabular}

In the table given above, capital letters of the miR site indicates the miRNA binding site of the $3^{\prime}-U T R$. Bold letters indicate SNPs identified in our study. $T S H R$, thyroid stimulating hormone receptor; SNP, single-nucleotide polymorphism; miRNA, microRNA; $\triangle \mathrm{G}$, binding energy; mer, nucleotide pairing. 
LINC00488, which is reported to be highly expressed in thyroid cancer cell lines, directly binds to miR-376a and downregulates its expression [28]. The rs17630128 variant was found to alter the binding affinity of hsa-miR-6858-3p.

The binding of TSH to TSHR induces angiogenesis by modulating vascular endothelial growth factor expression through cAMPmammalian target of rapamycin signaling [29]. In endurance athletes, the frequency of rs7144481 C-allele (wild-type allele) was reported to be higher than in controls, contributing to a high metabolic rate and better aerobic performance [30]. Through the regulation of gene expression, the rs7144481 SNP may decrease angiogenesis and/or the metabolic rate. Campo and his group found that rare allele carriers of rs7144481 in TSHR were at an increased risk of well-differentiated thyroid cancer [31].

\section{In silico predictions of function of the c. $1284 \mathrm{G}>\mathrm{C}$ (p.S398T) mutation in the TPO protein}

The docked structures of the wild-type and mutant structures with DUOX were saved for further structure-based analysis. The predicted wild-type and mutant structures were aligned using chimera to analyze the structural variation caused by the mutation. Various interactions between the wild-type and the mutant protein are tabulated and provided in Supplementary Table 1. The binding energies and dissociation constants for the wild-type and mutant complexes were also analyzed to reveal the functional alterations induced by the SNPs (Fig. 1). The TPO structures show a fluctuation in the root-mean-square deviation (RMSD) of $0.152 \AA$ and the in- teracting DUOX shows an RMSD of $1.091 \AA$. This shows that the SNP induces more structural variations in the binding protein DUOX than in TPO, which harbors the SNP. These structural variations induced in the binding complex alter the binding pattern which causes major variations in functional aspects of TPO. The mutant induces novel non-bonded hydrophobic interactions by the Ala263 residue, while there is a steep fall in the numbers of hydrophobic interactions by Trp200 and Leu267. A similar fall in the numbers of hydrogen-bonds involving main chain-main chain, main chain-side chain, and side chain-side chain patterns are observed. A steep fall in the number of ionic interactions by Arg198 with DUOX is observed in the mutant protein complex, whereas the aromatic-aromatic interactions and cation-pi interactions are retained. The complete list of the molecular interactions between the TPO wild-type and mutant proteins and the DUOX protein are given in Supplementary Table 1. These altered interactions further decrease the binding energies. The wild-type complex showed a $\Delta \mathrm{G}$ of $-15 \mathrm{kcal} / \mathrm{mol}$ and a dissociation constant $\left(\mathrm{K}_{\mathrm{d}}\right)$ of $7.2 \mathrm{E}^{-12} \mathrm{M}$, and the mutant showed a $\Delta \mathrm{G}$ of $-13.8 \mathrm{kcal} / \mathrm{mol}$ and a $\mathrm{K}_{\mathrm{d}}$ of $7.0 \mathrm{E}^{-11}$ $M$. These binding energies and dissociation constants further show that the mutant complex is less stable and dissociates more easily than the wild-type protein. The in silico analysis of p.S398T revealed that the mutant protein complex is less stable and it may be deleterious.

TPO and TG play a pivotal role in the biosynthesis of thyroid hormones by supplying hydrogen peroxide $\left(\mathrm{H}_{2} \mathrm{O}_{2}\right)$ and by serving as an iodine acceptor [32]. TPO protein activity hinges on the

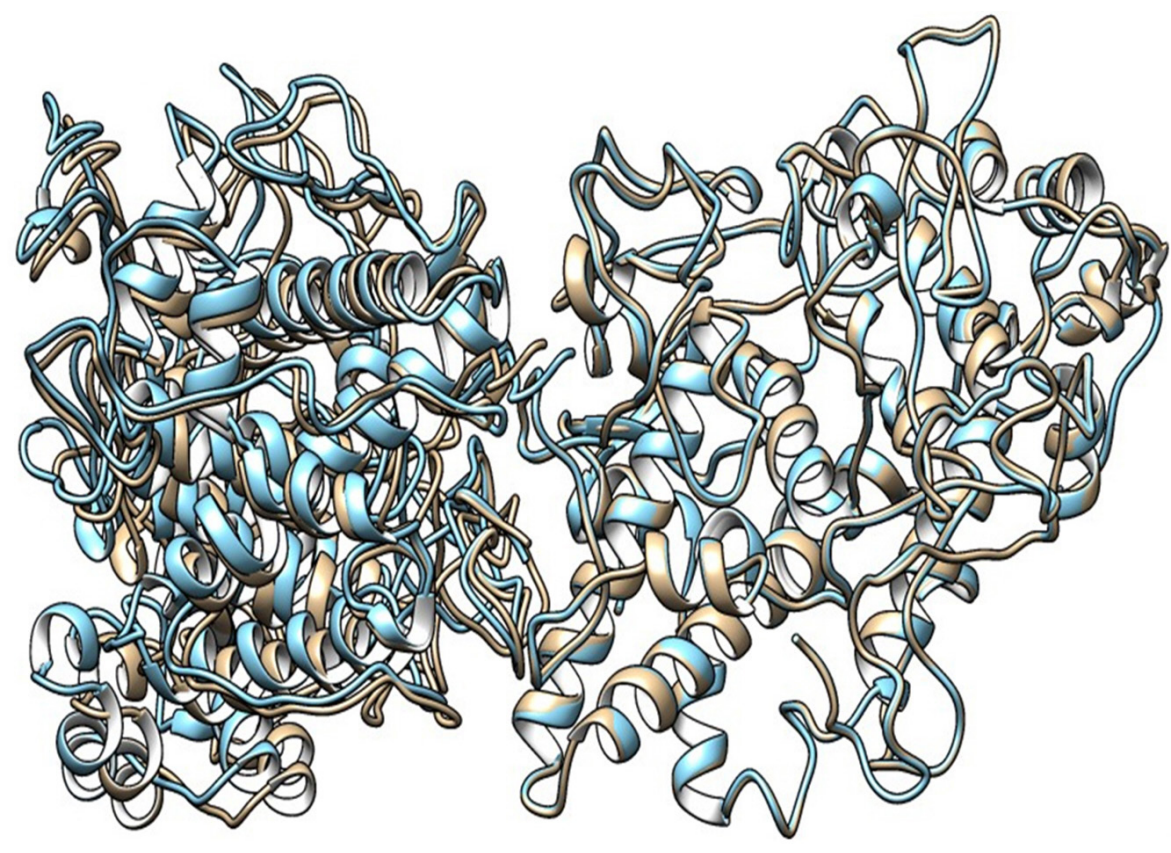

Fig. 1. Alignment showing the wild-type thyroid peroxidase (TPO) and mutant TPO with dual oxidase. The wild-type complex is shown in blue and the mutant complex is shown as mutant TPO. Deviation in the complex is shown by the non-alignment of the structures. 
proper folding and insertion of the membrane, as well as a complete catalytic site with the heme-binding region, which is encoded by exons 8,9 , and 10 [33]. TPO gene-inactivating mutations lead to iodide organification defect (IOD), which is either partial (PIOD) or total (TIOD) depending on the mutation type and position. IOD is diagnosed by a positive perchlorate discharge test (PDT) [34]. Turkkahraman et al. [35] performed PDT after the fourth week of the $\mathrm{LT}_{4}$ period. They found that one patient (variant-p. S398T) had PIOD (24.4\%) with positive PDT (normal range, $<10 \%)$. They suggested that PIOD should be considered in infants with permanent subclinical hypothyroidism [35].

TPO c.1284G > C (p.S398T) is located in the eighth exon of $T P O$, which is part of the heme-binding catalytic site of TPO. Hence, the decreased interaction stability results in impaired binding to heme and affects its interaction with iodide and TG [36]. Guriaet al. demonstrated that p.A373S, p.S398T, and p.T725P had damaging effects on TPO mRNA expression and protein activity [37]. Furthermore, they measured wild-type and mutant enzyme activity using an iodide and guaiacol assay and confirmed that the p.A373S and p.T725P mutants were more damaging than the p.S398T mutant [37]. Begum et al. performed an analysis of quantum mechanics/molecular mechanics and molecular dynamics on p.A373S, p.S398T, and p.T725P. This molecular docking study showed that the full-length TPO mutant p.S398T structure interacted with all the crucial amino acids in the catalytic site of the TPO protein. Begum et al. [38] concluded that the mutant variants p.A373S, p.S398T, and p.T725P were involved in Bangladeshi patients with thyroid dyshormonogenesis, and their molecular docking- based study showed that the three mutant variants had damaging effects on the activity of the TPO protein. In our study, the protein structure of TPO, along with that of DUOX1, was crystallized, and the mutant protein complex was predicted to be less stable, which may have an effect on the function of the protein. The mutant protein p.S398T was analyzed for binding energies and dissociation constants for wild-type versus mutant complexes, with results of $\Delta \mathrm{G}-15$ vs. $-13.8 \mathrm{kcal} / \mathrm{mol}$ and $\mathrm{K}_{\mathrm{d}} 7.2 \mathrm{E}^{-12}$ vs. $7.0 \mathrm{E}^{-11} \mathrm{M}$, respectively. The RMSD of the TPO protein structure is high (1.091 A) when it interacted with the DUOX protein, which illuminates the fact that the variant induced more structural variations in the binding protein DUOX than in TPO, which harbored the variant.

\section{In silico predictions of function of c.1999 G $>$ A (p.G653D) and c.6036 C $>\mathrm{T}$ (p.R1999W) in the TG protein}

No three-dimensional structures are available for any TG regions [39]. Three-dimensional structural folding of a long-chain amino acid sequence was seen as a complex problem in the past [40]. Due to high molecular weight of TG and the lack of its crystal structure, in silico studies on TG have not been conducted to date. The recent elucidation of its crystal structure (PDB ID: 6SCJ) [23] facilitated the in silico exploration of $T G$ variants. The predicted wild-type and mutant (p.G653D and p.R1999W) structures of TG proteins were aligned using the PyMol software [24] to analyze the effects of structural variation caused by the mutation in the surrounding residues within $4 \AA$.

\section{Analysis of the effect of the p.G653D mutation}

The analysis of the structure of the p.G653D mutant of TG showed that the mutation of Gly653 to Asp653 formed hydrogen-bonding interactions with the surrounding residue Ser990 (2.4 $)$ as shown in Fig. 2, resulting in altered TG protein structure (Supplementary Video 1). The SAAMBE-3D prediction showed that the mutation destabilized the TG protein, with a $\Delta \mathrm{G}$ value of $0.24 \mathrm{kcal} / \mathrm{mol}$.

Next, the effect of the mutation on the adjacent residues was investigated using the DynaMut server [25]. The mutant Asp653 formed a weak polar van der Waals bond with the residues Gly555 and Ser885, polar proximal interactions with Gln576, and amide-amide interactions with Ser885, as shown in Fig. 3.

\section{Analysis of the effect of the p.R1999W mutation}

Next, the analysis of the mutant (p.R1999W) TG structures showed that the mutation of Arg1999 to Trp1999 formed hydrogen-bonding interactions with the surrounding residues, such as

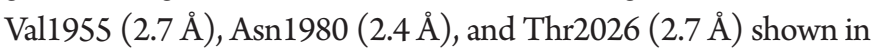
Fig. 4 (Supplementary Video 2). This shows that the mutation of p.R1999W resulted in bonding interactions with the surrounding residues, which may affect the structure-function relationship of the TG protein. The effect of the p.R1999W mutation on protein stability was further assessed using the SAAMBE-3D server. The SAAMBE-3D prediction showed that the mutation destabilized the $T G$ protein and the $\Delta G$ value was $0.79 \mathrm{kcal} / \mathrm{mol}$.

The analysis of bonding and non-bonding interactions shows that the p.R1999W mutation results in non-bonding interactions with the residues; for instance, Trp1999 shows van der Waals clashes with Glu1820 and Thr1845, hydrogen-bond and van der Waals clashes with Glu1818, hydrophobic proximal clashes with Val1805 and Leu1780, hydrogen-bond proximal interactions with Ala1825, van der Waals clashes with Asn1803, and carbon-pi interactions with Val1805 (Fig. 5).

These altered interactions due to the p.G653D and p.R1999W mutations decrease the binding energies; here, the mutation of p.R1999W shows a more destabilizing effect on the TG protein due to more hydrogen-bonding interactions (Fig. 4), van der Waals and carbon-pi interactions (Fig. 5), and a higher binding energy $(\Delta \mathrm{G}=$ $0.79 \mathrm{kcal} / \mathrm{mol})$ compared to the p.G653D mutation $(\Delta \mathrm{G}=0.24$ 


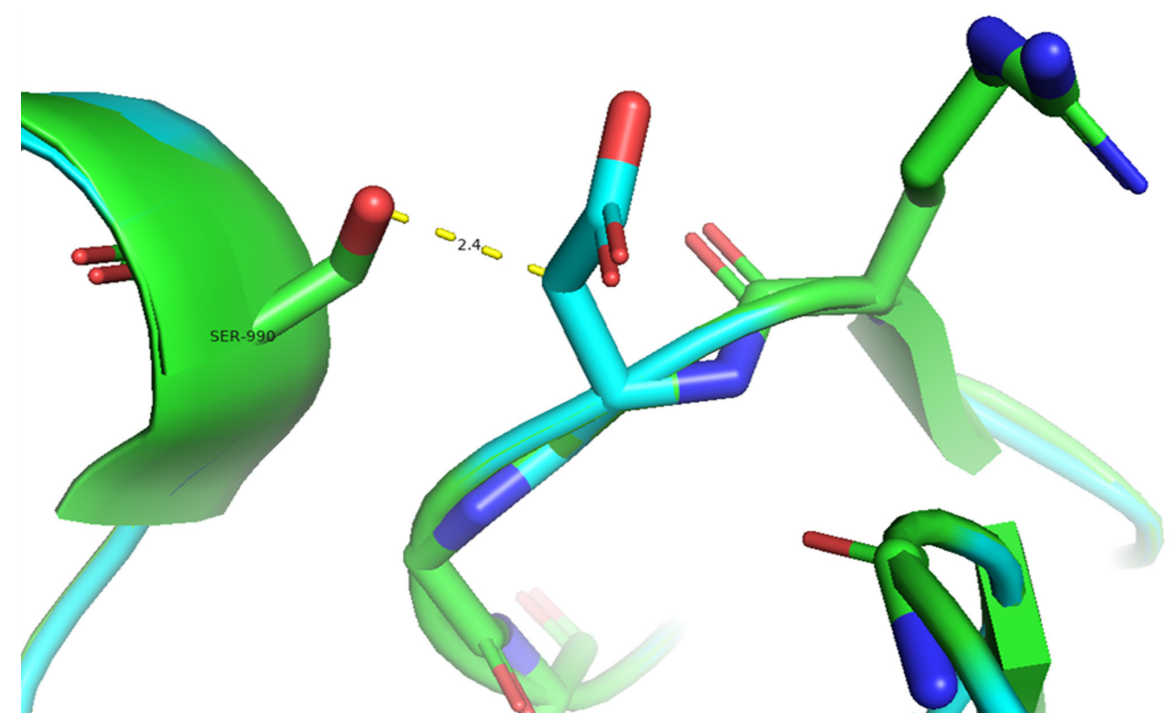

Fig. 2. An in silico analysis shows overlapping images of the wild-type (green) and mutant G653D (cyan) thyroglobulin protein. Here, the mutant residue Asp653 is shown in a stick model with hydrogen-bonding interactions with the Ser990 residue.

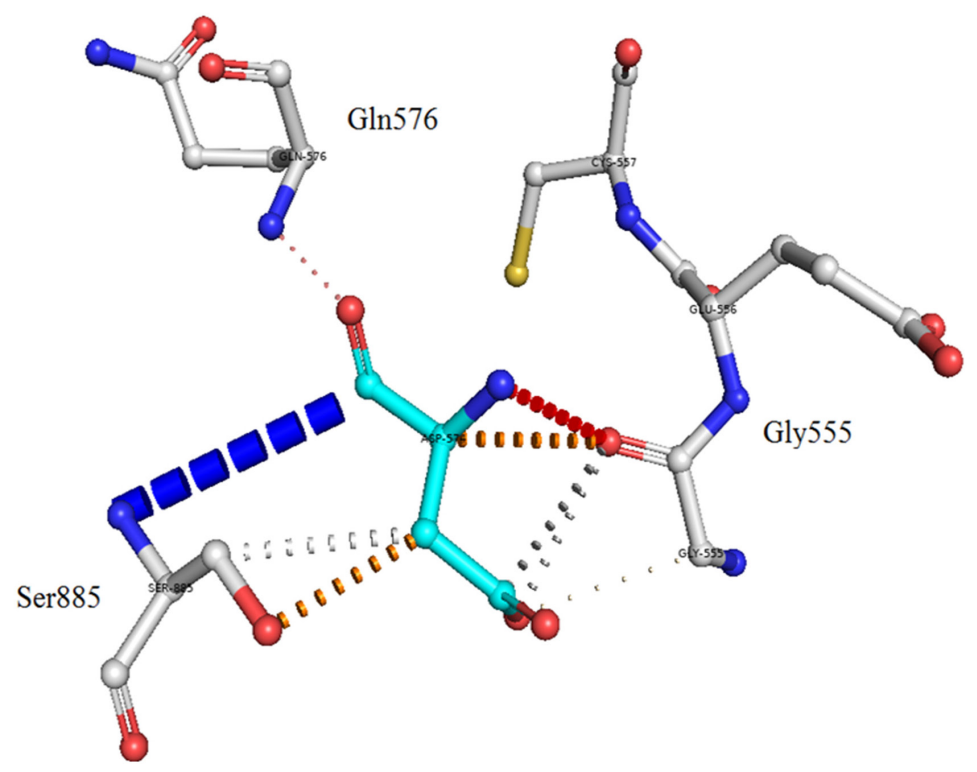

Fig. 3. An in silico analysis shows the mutant residue Asp653 colored in cyan, which is also represented as sticks alongside with the surrounding residues colored in white, which are involved in any type of interactions. The mutant thyroglobulin protein shows the weak polar van der Waals clashes with the residues Gly555 and Ser885, polar proximal interactions with Gln576, and amide-amide interactions with Ser885.

$\mathrm{kcal} / \mathrm{mol})$. These structural and energetic analyses show that the mutants are less stable and affect the structure-functional relationship of the human TG protein.

Aljouie et al. studied synonymous and nonsynonymous variants such as rs76672487 in the ABCC2 gene and rs2069548 (p.G653D) in the $T G$ gene. According to the Human Protein Atlas, these two genes are cancer-related genes. The variant p.G653D was ranked fourth of the selected variants using the chi-square test from the top SNPs in the glioblastoma multiforme +1000 genome database. The nearby tissue was enriched in this rare variant. This variant affects cancer susceptibility by suppressing mRNA expression (transcription and translation) [41]. Autoimmune thyroid diseases, including hyperthyroidism/Graves' disease and autoimmune hypothyroidism/Hashimoto's thyroiditis, are complex diseases caused by a malfunction in immune tolerance to self-thyroid antigens, such as TSHR, TPO, and TG [42]. Pyun et al. [43] identified epistasis between two polymorphic variants of the HSD17B4 and TG genes. One variant, p.R1999W (c.5995 C > T), in the TG gene was tested 


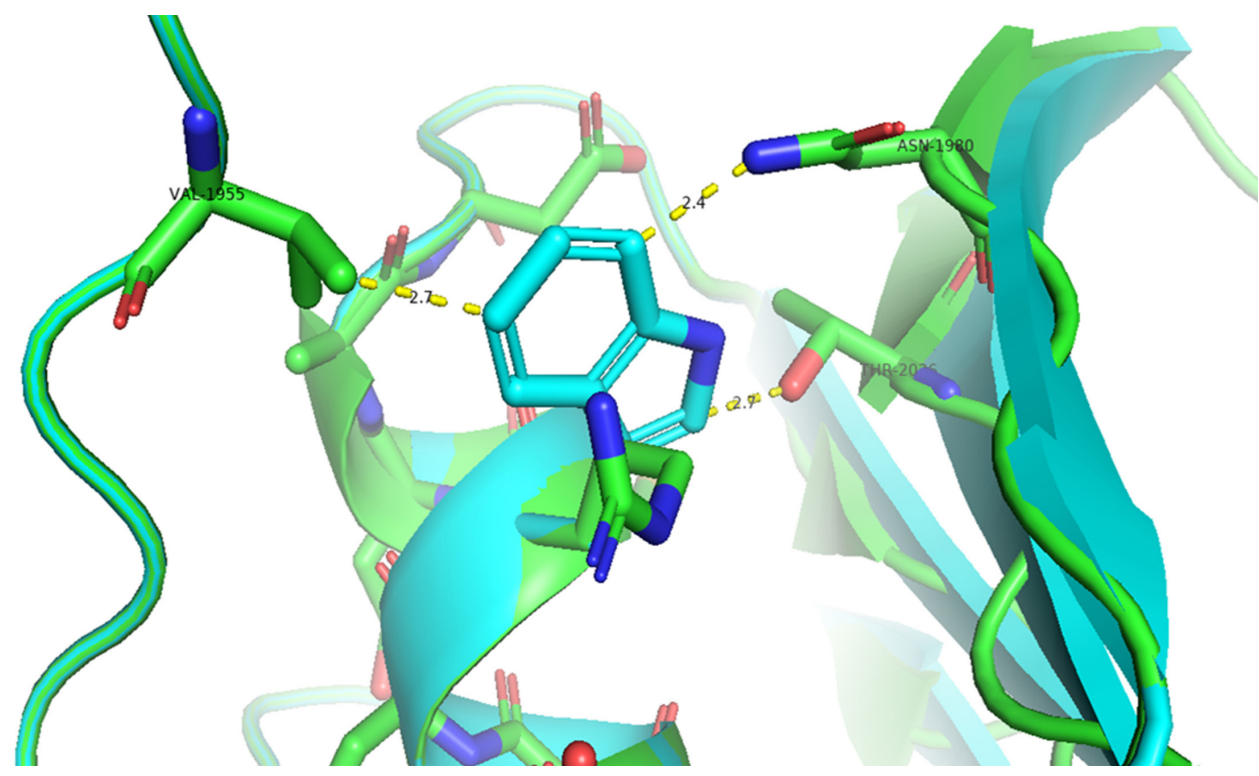

Fig. 4. An in silico analysis shows the overlapped images of wild-type (green) and mutant R1999W (cyan) thyroglobulin protein. The wildtype Arg1999 is shown in green and the mutant Trp1999 is shown in cyan. The mutant Trp1999 forms bonding interactions with Val1955, Asn1980, and Thr2026.
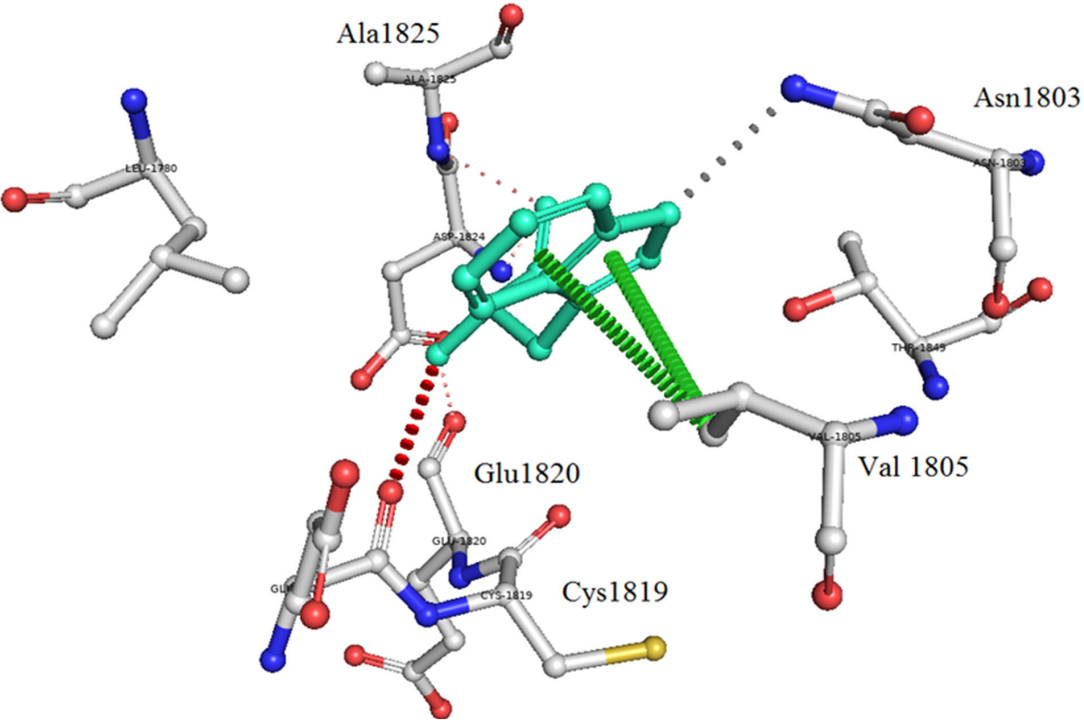

Fig. 5. An in silico analysis shows the mutant p.R1999W residue colored in light-green and also represented as sticks alongside with the surrounding residues colored in white, which are involved in any type of interactions. Here, Trp1999 shows van der Waals clashes with Glu1820 and Thr1845, hydrogen-bond and van der Waals clashes with Glu1818, hydrophobic proximal clashes with Val1805 and Leu1780, hydrogen-bond proximal interactions with Ala1825, van der Waals clashes with Asn1803, and carbon-pi interactions with Val1805.

for epistasis with the HSD17B4 variant p.T687I (c.2060 C > T). The combined effect of these variants was significantly associated with premature ovarian failure, although these variants alone showed no significant association. Ban et al. [44] performed case-control association studies for 14 discovered $T G$ variants in 285 autoimmune thyroid disease patients and 150 controls. One variant cluster (p.S753A \& p.P797P in exon 10 and p.M1027V in exon 12) [44] and the exon 33 variant p.R1999W showed significant associations with patients suffering from autoimmune thyroid disease $[44,45]$ in the United States [44], but were not associated with patients in the United Kingdom suffering from the same disease [42]. The combination of these variants conferred susceptibility to autoimmune thyroid disease. Ban et al. [44] analyzed genegene interactions between p.R1999W in the TG gene and HLA- 
$D R 3$. The variant (p.R1999W) showed promising results for interaction with HLA-DR3 in conferring susceptibility to Graves' disease (odds ratio, 6.1) [44].

Although there are no reports depicting the direct association of p.G653D and p.R1999W variants of the TG protein with $\mathrm{CH}$, several recent studies have illuminated the role of TG variants in the etiology of $\mathrm{CH}$. These studies illustrate $\mathrm{TG}$ as one of the primary candidate genes to be evaluated in $\mathrm{CH}$ patients [46-49]. In our study, we investigated the effect of mutations such as p.G653D and p.R1999W, which were analyzed using computational structural biology methods, including mutant model building for the TG protein and structural analysis of interaction networks such as hydrogen-bonding. A further analysis of binding energies using the SAAMBE-3D server demonstrated that the p.G653D and p. R1999W mutations showed a protein destabilizing effect, which revealed that these SNPs may be deleterious and affect the TG protein structure-function relationship.

In conclusion, in silico studies revealed that SNPs in the $3^{\prime}$-UTR region altered the binding affinity of various miRs, thus influencing the expression of thyroid-associated genes. In this study, analyses of the computational protein-protein interactions and the binding energies of the p.S398T mutation in the TPO gene showed that the mutant protein complex was less stable than the wild-type complex, implying that this SNP may be deleterious. The altered variants p.G653D and p.R199W decreased the binding energies and contributed to a destabilizing effect on the TG protein.

\section{ORCID}

Yedukondalu Kollati: https://orcid.org/0000-0002-1851-3213

Radha Rama Devi Akella: https://orcid.org/0000-0002-4108-6213 Shaik Mohammad Naushad: https://orcid.org/0000-0001-89529581

Rajesh K. Patel: https://orcid.org/0000-0002-7210-6168

G. Bhanuprakash Reddy: https://orcid.org/0000-0003-4787-3944

Vijaya R. Dirisala: https://orcid.org/0000-0002-9433-2780

\section{Authors' Contribution}

Conceptualization: VRD, SMN, RRDA, GBR. Data curation: YK, RRDA. Formal analysis: YK, RKP. Funding acquisition: VRD. Methodology: YK, SMN, VRD. Writing - original draft: YK, SMN. Writing - review \& editing: RRDA, RKP, GBR, VRD.

\section{Conflicts of Interest}

No potential conflict of interest relevant to this article was reported.

\section{Acknowledgments}

This work was partly supported by a grant from DST-SERB, Government of India (ECR/2016/00304). The authors specially thank Dr. Pasumarthi NBS Srinivas, Dr. Hari Krishna K, Dr. Bajarang Vasant Kumbhar, Anusha Puvvada, Uma Maheshwar P for their support during the investigation.

\section{Supplementary Materials}

Supplementary data can be found with this article online at http:// www.genominfo.org.

\section{References}

1. Agrawal P, Philip R, Saran S, Gutch M, Razi MS, Agroiya P, et al. Congenital hypothyroidism. Indian J Endocrinol Metab 2015; 19:221-227.

2. Ahmad N, Irfan A, Al Saedi SA. Congenital hypothyroidism: screening, diagnosis, management, and outcome.J Clin Neonatol 2017;6:64-70.

3. ICMR Task Force on Inherited Metabolic Disorders. Newborn screening for congenital hypothyroidism and congenital adrenal hyperplasia. Indian J Pediatr 2018;85:935-940.

4. Kollati Y, Ambati RR, Reddy PN, Kumar NS, Patel RK, Dirisala VR. Congenital hypothyroidism: facts, facets and therapy. Curr Pharm Des 2017;23:2308-2313.

5. Ramesh BG, Bhargav PR, Rajesh BG, Devi NV, Vijayaraghavan R, Varma BA. Genotype-phenotype correlations of dyshormonogenetic goiter in children and adolescents from South India. Indian J Endocrinol Metab 2016;20:816-824.

6. Kota SK, Modi K, Kumaresan K. Elevated thyroid stimulating hormone in a neonate: drug induced or disease? Indian J Endocrinol Metab 2011;15(Suppl 2):S138-S140.

7. Lee CC, Harun F, Jalaludin MY, Heh CH, Othman R, Kang IN, et al. Variable clinical phenotypes in a family with homozygous c.1159G > A mutation in the thyroid peroxidase gene. Horm Res Paediatr 2014;81:356-360.

8. Sironi M, Menozzi G, Riva L, Cagliani R, Comi GP, Bresolin N, et al. Silencer elements as possible inhibitors of pseudoexon splicing. Nucleic Acids Res 2004;32:1783-1791.

9. Dhanoa JK, Sethi RS, Verma R, Arora JS, Mukhopadhyay CS. Long non-coding RNA: its evolutionary relics and biological implications in mammals: a review. J Anim Sci Technol 2018;60:25.

10. Christopher AF, Kaur RP, Kaur G, Kaur A, Gupta V, Bansal P. MicroRNA therapeutics: discovering novel targets and developing specific therapy. Perspect Clin Res 2016;7:68-74. 
11. Popp NA, Yu D, Green B, Chew EY, Ning B, Chan CC, et al. Functional single nucleotide polymorphism in IL-17A 3' untranslated region is targeted by miR-4480 in vitro and may be associated with age-related macular degeneration. Environ $\mathrm{Mol}$ Mutagen 2016;57:58-64.

12. Kollati Y, Akella RR, Naushad SM, Borkar D, Thalla M, Nagalingam $S$, et al. Newborn screening and single nucleotide variation profiling of TSHR, TPO, TG and DUOX2 candidate genes for congenital hypothyroidism. Mol Biol Rep 2020;47:7467-7475.

13. Bhattacharya A, Ziebarth JD, Cui Y. PolymiRTS Database 3.0: linking polymorphisms in microRNAs and their target sites with human diseases and biological pathways. Nucleic Acids Res 2014;42:D86-D91.

14. Liu W, Wang X. Prediction of functional microRNA targets by integrative modeling of microRNA binding and target expression data. Genome Biol 2019;20:18.

15. Agarwal V, Bell GW, Nam JW, Bartel DP. Predicting effective microRNA target sites in mammalian mRNAs. Elife 2015;4: e05005.

16. Kanoria S, Rennie W, Liu C, Carmack CS, Lu J, Ding Y. STarMir tools for prediction of microRNA binding sites. Methods Mol Biol 2016;1490:73-82.

17. Yang J, Yan R, Roy A, Xu D, Poisson J, Zhang Y. The I-TASSER Suite: protein structure and function prediction. Nat Methods 2015;12:7-8.

18. Pettersen EF, Goddard TD, Huang CC, Couch GS, Greenblatt DM, Meng EC, et al. UCSF chimera: a visualization system for exploratory research and analysis. J Comput Chem 2004;25: 1605-1612.

19. Kozakov D, Hall DR, Xia B, Porter KA, Padhorny D, Yueh C, et al. The ClusPro web server for protein-protein docking. Nat Protoc 2017; 12:255-278.

20. Tina KG, Bhadra R, Srinivasan N. PIC: protein interactions calculator. Nucleic Acids Res 2007;35:W473-W476.

21. Vangone A, Bonvin AM. Contacts-based prediction of binding affinity in protein-protein complexes. Elife 2015;4:e07454.

22. Pahari S, Li G, Murthy AK, Liang S, Fragoza R, Yu H, et al. SAAMBE-3D: Predicting Effect of Mutations on Protein-Protein Interactions. Int J Mol Sci 2020;21:2563.

23. Coscia F, Taler-Vercic A, Chang VT, Sinn L, O’Reilly FJ, Izore T, et al. The structure of human thyroglobulin. Nature 2020;578:627-630.

24. Delano WL. The PyMOL molecular graphics system, version 1.8. New York: Schrodinger, 2002.

25. Rodrigues CH, Pires DE, Ascher DB. DynaMut: predicting the impact of mutations on protein conformation, flexibility and stability. Nucleic Acids Res 2018;46:W350-W355.
26. Kollati Y, Akella RR, Naushad SM, Thalla M, Reddy GB, Dirisala VR. The rs 1991517 polymorphism is a genetic risk factor for congenital hypothyroidism. 3 Biotech 2020;10:285.

27. Marini F, Luzi E, Brandi ML. MicroRNA role in thyroid cancer development. J Thyroid Res 2011;2011:407123.

28. Xie F, Li L, Luo Y, Chen R, Mei J. Long non-coding RNA LINC00488 facilitates thyroid cancer cell progression through miR-376a-3p/PON2. Biosci Rep 2021;41:BSR20201603.

29. Balzan S, Del Carratore R, Nicolini G, Beffy P, Lubrano V, Forini F, et al. Proangiogenic effect of TSH in human microvascular endothelial cells through its membrane receptor. J Clin Endocrinol Metab 2012;97:1763-1770.

30. Ahmetov I, Kulemin N, Popov D, Naumov V, Akimov E, Bravy Y, et al. Genome-wide association study identifies three novel genetic markers associated with elite endurance performance. Biol Sport 2015;32:3-9.

31. Campo C, Kohler A, Figlioli G, Elisei R, Romei C, Cipollini M, et al. Inherited variants in genes somatically mutated in thyroid cancer. PLoS One 2017;12:e0174995.

32. Targovnik HM, Citterio CE, Rivolta CM. Iodide handling disorders (NIS, TPO, TG, IYD). Best Pract Res Clin Endocrinol Metab 2017;31:195-212.

33.Deladoey J, Pfarr N, Vuissoz JM, Parma J, Vassart G, Biesterfeld S, et al. Pseudodominant inheritance of goitrous congenital hypothyroidism caused by TPO mutations: molecular and in silico studies. J Clin Endocrinol Metab 2008;93:627-633.

34. Turkkahraman D, Alper OM, Pehlivanoglu S, Aydin F, Yildiz A, Luleci G, et al. Analysis of TPO gene in Turkish children with iodide organification defect: identification of a novel mutation. Endocrine 2010;37:124-128.

35. Turkkahraman D, Alper OM, Aydin F, Yildiz A, Pehlivanoglu S, Luleci $G$, et al. Final diagnosis in children with subclinical hypothyroidism and mutation analysis of the thyroid peroxidase gene (TPO). J Pediatr Endocrinol Metab 2009;22:845-851.

36. Rivolta CM, Moya CM, Esperante SA, Gutnisky VJ, Varela V, Targovnik HM. The thyroid as a model for molecular mechanisms in genetic diseases. Medicina (B Aires) 2005;65:257-267.

37. Guria S, Bankura B, Balmiki N, Pattanayak AK, Das TK, Sinha A, et al. Functional analysis of thyroid peroxidase gene mutations detected in patients with thyroid dyshormonogenesis. Int J Endocrinol 2014;2014:390121.

38. Begum MN, Islam MT, Hossain SR, Bhuyan GS, Halim MA, Shahriar I, et al. Mutation sectrum in TPO gene of Bangladeshi patients with thyroid dyshormonogenesis and analysis of the effects of different mutations on the structural features and functions of TPO protein through in silico approach. Biomed Res Int 2019;2019:9218903. 
39. Targovnik HM, Citterio CE, Rivolta CM. Thyroglobulin gene mutations in congenital hypothyroidism. Horm Res Paediatr 2011;75:311-321.

40. Haddad Y, Adam V, Heger Z. Ten quick tips for homology modeling of high-resolution protein 3D structures. PLoS Comput Biol 2020; 16:e1007449.

41. Aljouie A, Schatz M, Roshan U. Machine learning based prediction of gliomas with germline mutations obtained from whole exome sequences from TCGA and 1000 Genomes Project. In: The 3rd International Conference on Intelligent Computing in Data Sciences (ICDS), 2019 Oct 28-30, Marrakech, Morocco.

42. Collins JE, Heward JM, Howson JM, Foxall H, Carr-Smith J, Franklyn JA, et al. Common allelic variants of exons 10, 12, and 33 of the thyroglobulin gene are not associated with autoimmune thyroid disease in the United Kingdom. J Clin Endocrinol Metab 2004;89:6336-6339.

43. Pyun JA, Kim S, Cha DH, Ko JJ, Kwack K. Epistasis between the HSD17B4 and TG polymorphisms is associated with premature ovarian failure. Fertil Steril 2012;97:968-973.

44. Ban Y, Greenberg DA, Concepcion E, Skrabanek L, Villanueva R, Tomer Y. Amino acid substitutions in the thyroglobulin gene are associated with susceptibility to human and murine autoimmune thyroid disease. Proc Natl Acad Sci U S A 2003;100:15119-15124.

45. Zhang ML, Zhang DM, Wang CE, Chen XL, Liu FZ, Yang JX. Association between thyroglobulin polymorphisms and autoimmune thyroid disease: a systematic review and meta-analysis of case-control studies. Genes Immun 2019;20:484-492.

46. Raef H, Al-Rijjal R, Al-Shehri S, Zou M, Al-Mana H, Baitei EY, et al. Biallelic p.R2223H mutation in the thyroglobulin gene causes thyroglobulin retention and severe hypothyroidism with subsequent development of thyroid carcinoma. J Clin Endocrinol Metab 2010;95:1000-1006.

47. Hu X, Chen R, Fu C, Fan X, Wang J, Qian J, et al. Thyroglobulin gene mutations in Chinese patients with congenital hypothyroidism. Mol Cell Endocrinol 2016;423:60-66.

48. Santos-Silva R, Rosario M, Grangeia A, Costa C, Castro-Correia $\mathrm{C}$, Alonso I, et al. Genetic analyses in a cohort of Portuguese pediatric patients with congenital hypothyroidism. J Pediatr Endocrinol Metab 2019;32:1265-1273.

49. Tanaka T, Aoyama K, Suzuki A, Saitoh S, Mizuno H. Clinical and genetic investigation of 136 Japanese patients with congenital hypothyroidism. J Pediatr Endocrinol Metab 2020;33:691-701. 